\title{
Noncompaction Phenotype, But What is Cause and is the Diagnosis Correct?
}

\section{(D) Onur Akhan}

Bilecik State Hospital, Clinic of Cardiology, Bilecik, Turkey

\begin{abstract}
Objectives: The study aims to reveal different groups of left ventricular (LV) noncompaction (LVNC) by evaluating patients who have undergone cardiac magnetic resonance imaging for the confirmation of diagnosis and also to examine detailed features of cardiac magnetic resonance imaging. Noncompaction cardiomyopathy is a rare myocardial pathology characterized by increased trabeculation. Diagnosis requires echocardiography-and cardiac magnetic resonance-based quantitative indexes measuring ratios of noncompacted and compacted layers of the left ventricle. Although LVNC can be seen as an isolated entity, it may also be a feature of several cardiac and noncardiac disorders.
\end{abstract}

Materials and Methods: Our study is a retrospective cohort study. Cardiac magnetic resonance imaging performed between the years of 2006 and 2018 in our radiology department were analyzed with the keyword "noncompaction". A total of 64 imaging were examined regarding diagnostic criteria, hypertrabeculated areas, LV ejection fraction values and comorbidities. Mortality and cardiac operation outcomes were also investigated.

Results: In our study population, 38 (\%59) of 64 patients were men. The mean age was 41 years in an age range of 18 73 years. Hypertrabeculations were prominently seen in the left ventricle lateral wall followed by the apex and inferior wall. Noncompacted and compacted myocardial layers thickness ratio was measured and averaged around $2.9(2.2$ -4) at the site of maximal wall thickness. Forty-eight reports had also data about LV ejection fraction values and the mean value was 35 (17-69). Regarding comorbidity, coronary artery disease, primary valve disease, connective tissue disease, duchenne muscular dystrophy, neurofibromatosis type 1, peripartum cardiomyopathy, and congenital heart anomalies were observed. Totally 38 patients were diagnosed with noncompaction cardiomyopathy. Also, biventricular noncompaction was observed in three patients and isolated right ventricle noncompaction was observed in one patient.

Address for Correspondence: Onur Akhan, Bilecik State Hospital, Clinic of Cardiology, Bilecik, Turkey

Phone: +90 5355943355 e-mail: akhanonur@gmail.com ORCID: orcid.org/0000-0003-4440-9599

Received: 14.04.2020 Accepted: 01.05.2020

Cite this article as: Akhan O. Noncompaction Phenotype, But What is Cause and is the Diagnosis Correct? EJCM 2020;8(2):85-90. DOI: 10.32596/ejcm.galenos.2020.03.013

Presented in: The manuscript was presented as a poster presentation at EuroCMR Congress 2019 in Venice/Italy on May 3, 2019 (Palazza del cinema - Venice) (EUD ID 571156). 


\begin{abstract}
Regarding cardiac operation endpoints, it was observed that cardiac resynchronization therapy-defibrillator implantation was performed in six patients, LV assist device implantation in five patients, and heart transplantation in three patients. Four patients were exitus concerning mortality.
\end{abstract}

Conclusion: The LVNC remains subject to controversy

\section{Introduction}

Noncompaction is a ventricular wall anatomy pathology consisting of increased trabeculation, a thin compact layer, and deep intertrabecular cavities ${ }^{(1)}$.

Normally, in the early systolic ejection phase, along with contraction, trabeculae act as a mechanical lever ${ }^{(2,3)}$. According to embryogenic hypothesis, the endomyocardial layer cannot be compacted in the early embryonic period in left ventricular (LV) noncompaction (LVNC) ${ }^{(2)}$. However, this hypothesis cannot explain the reversible LVNC situations which can be seen as a result of physiological adaptation such as pregnancy, athletes, sickle cell anemia, and chronic kidney failure ${ }^{(3)}$. Depending on the pathological loading conditions, the related situation may also occur as a result of pathological remodeling ${ }^{(4,5)}$.

In 1980, cardiomyopathy was defined as "heart muscle diseases of unknown cause" by the World Health Organization (WHO) to distinguish cardiomyopathy from other cardiovascular entities ${ }^{(6)}$. In 1995, this definition was arranged as myocardial diseases associated with cardiac dysfunction. Noncompaction cardiomyopathy (NCCM) term is defined also as a myocardial disorder characterized by increased trabeculations linked with deep recesses that communicate with the LV cavity ${ }^{(7)}$.

In recent years, asymptomatic LVNC cases are increasing ${ }^{(8)}$. Therefore, multi-parameter [electrocardiography (ECG), echocardiography, stress test, 24-hour Holter ECG evaluation, and cardiac magnetic resonance (CMR) imaging] evaluation should be performed ${ }^{(9)}$. The diagnostic criteria are due to lack of consensus on its etiology, pathophysiology, diagnosis and management. There is a requirement for a consensus about diagnostic imaging modalities, and accompanying conditions about noncompaction.

Keywords: Noncompaction, cardiomyopathy, cardiac magnetic resonance imaging

mainly based on the measurement of noncompacted (NC) to compacted (C) layer thickness ratio, trabeculation masses, and volumes. The number of NC segments can provide additional information about the spread of LVNC. CMR imaging is superior to echocardiography in the definition of non-compacted myocardial tissue, but there are still some difficulties with the sensitivity and specificity ${ }^{(2)}$.

As stated above, echocardiography and CMR imaging are at the forefront in the diagnosis of LVNC. In the CMR imaging, it was accepted as the diagnostic criteria that the trabecular mass constituted at least $20 \%$ of the myocardial mass, and $\mathrm{NC}$ to $\mathrm{C}$ layer thickness ratio of $\geq 2.3$ at enddiastole in at least two short-axis CMR slices. There are also studies related to the determination of regional wall movements and late gadolinium enhancement (LGE) ${ }^{(10)}$.

\section{Aim}

The study aims to reveal different groups of LVNC with the evaluation of patients who have undergone CMR imaging for the confirmation of diagnosis and also to examine detailed features of CMR reports.

\section{Materials and Methods}

Our study is a retrospective cohort study. This study was approved by the local ethical committee at our university (date: 8/8/17, decision no: 17-6/12).

\section{Patient Selection}

We analyzed the CMR imagings between January 1, 2006 [the year that NCCM was considered as a primary 
cardiomyopathy by American Heart Association (AHA) ${ }^{(11)}$ ] and December 31, 2018 in our radiology department with the keyword "noncompaction". A total of 64 CMR imagings were examined regarding diagnostic criteria, hypertrabeculated areas, LV ejection fraction (LVEF) values, and accompanying disease.

\section{Patient Outcomes}

After the evaluation of these reports, we also investigated these patients concerning mortality and cardiac interventions/operations endpoints. Morbidity and cardiac operation information was obtained from the hospital information system. In addition, patients and their relatives were contacted by phone, and information about the cardiac interventions/operations performed at other hospitals, if any, was also obtained. Mortality information was obtained from our country's death notification system.

\section{Statistical Analysis}

After the evaluation of nearly 12 years of reports, information was obtained. However, not all reports were standardized in these 12 years. An adequate data could not be obtained for statistical analysis due to the deficit of parameters related to NCCM diagnosis, other values like LVEF in the reports, and differences between operators.

\section{Results}

Baseline demographic and CMR characteristics of the patients are listed in Table 1. Representative CMR image from a patient with LVNC are also shown in Figures 1 and 2.

Table 1. Baseline Characteristics

\begin{tabular}{l|l}
\hline Characteristics & $\mathbf{n = 6 4}$ \\
\hline Age (mean-range) & $41(18-73)$ \\
\hline Male gender, n (\%) & $38(59 \%)$ \\
\hline LVEF (mean-range)* & $35(17-69)$ \\
\hline Maximum NC/C ratio (mean-range)* & $2.9(2.2-4)$ \\
\hline Number of NC segments (mean-range) & $4.8(3-6)$ \\
\hline $\begin{array}{l}\text { LVEF: Left ventricular ejection fraction, NC: Noncompaction, C: } \\
\text { Compacted, } n: \text { Number } \\
\text { *In some reports, there were insufficient data }\end{array}$ \\
\hline
\end{tabular}

After the evaluation of CMR reports, people had advanced imaging for differential diagnosis of LVNC. In our study population, 38 (59\%) of 64 patients were men. The mean age was 41 years in an age range of $18-73$ years.

Hypertrabeculation was prominently seen in LV lateral wall followed by the apex and inferior wall. The ratio of thickness of $\mathrm{NC}$ and $\mathrm{C}$ myocardial layers at the site of maximal wall was measured and averaged around 2.9 (2.24). In 48 of the current CMR reports, information about LVEF was available, and the mean value was 35 (17-69).

Regarding comorbidity, coronary artery disease, primary valve disease, connective tissue disease, duchenne muscular dystrophy, neurofibromatosis type 1, peripartum cardiomyopathy, and congenital heart anomalies were observed. Considering the definition of cardiomyopathy, which is "heart muscle diseases of unknown cause", after the exclusion of accompanying pathologies, physiologic and pathologic adaptation processes, the remaining cases were evaluated according to NCCM diagnostic criteria. Four of the remaining cases did not meet the diagnostic criteria, and six had insufficient data for the diagnosis. Totally 38 patients were diagnosed with NCCM. In addition, biventricular $\mathrm{NC}$ was observed in three patients and isolated right ventricle $\mathrm{NC}$ was observed in one patient.

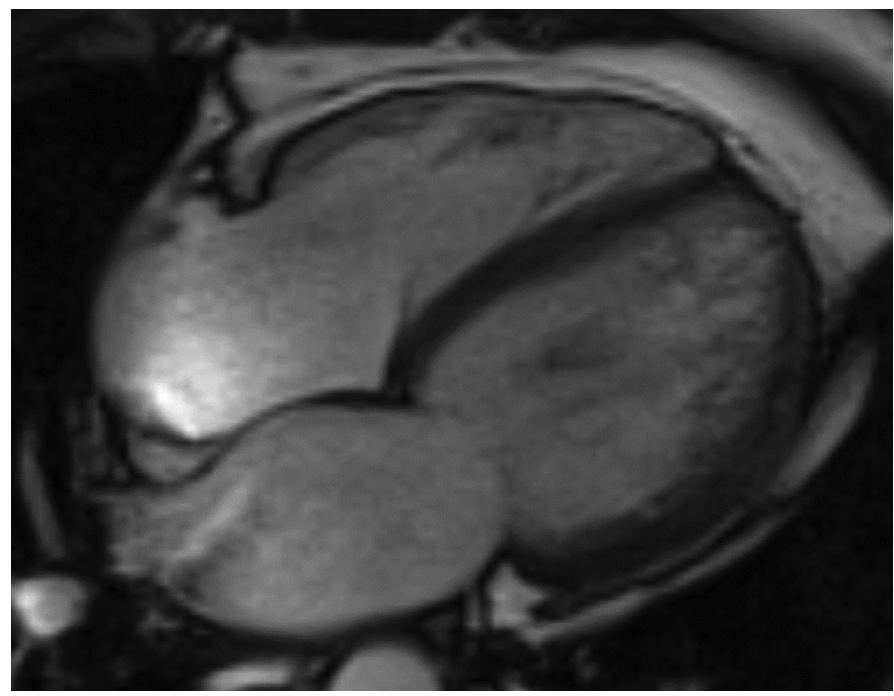

Figure 1. Representative CMR image from a patient with LVNC CMR: Cardiac magnetic resonance, LVNC: Left ventricular noncompaction 
It is controversial whether the NC pattern seen in peripartum cardiomyopathy is reversible and whether peripartum cardiomyopathy is a heterogeneous condition. Therefore, peripartum cardiomyopathy was not included in NCCM.

Accompanying disorders to LVNC patterns other than NCCM are listed in Table 2.

Regarding cardiac interventions/operations endpoints, it was observed that cardiac resynchronization therapydefibrillator implantation was performed in six patients, LV assist device (LVAD) implantation in five patients, and heart transplantation in three patients. It was also observed that two of these patients underwent both LVAD implantation and subsequent heart transplantation. Death notification system data of our country were used for mortality information and it was learned that four patients were dead.

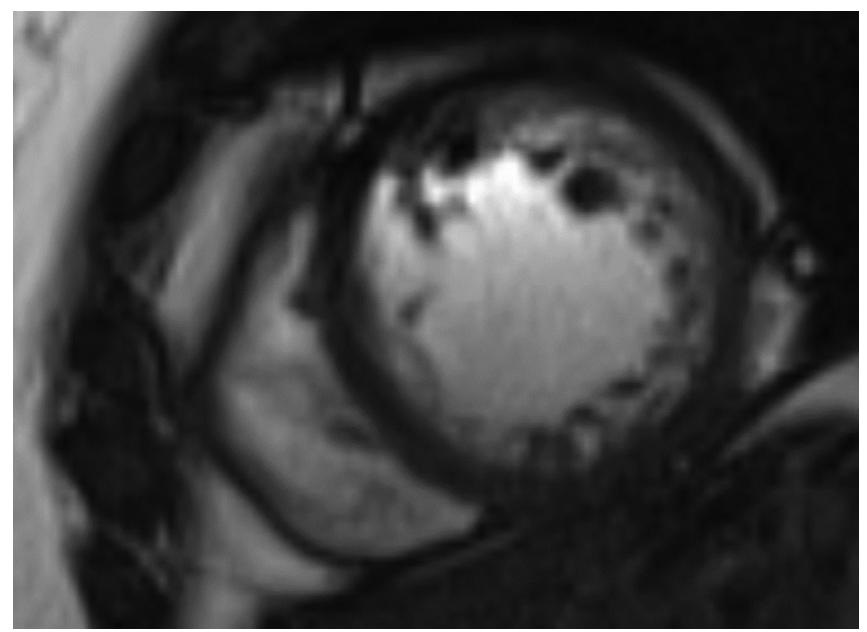

Figure 2. Representative CMR image from a patient with LVNC CMR: cardiac magnetic resonance, LVNC: Left ventricularnoncompaction

Table 2. Accompanying Disorders

\begin{tabular}{|l|l|}
\hline Comorbidities & $\mathbf{n}$ \\
\hline Coronary Artery Disease & 5 \\
\hline Primary Valve Disease & 2 \\
\hline Connective Tissue Disorder & 1 \\
\hline Duchenne Muscular Dystrophy & 1 \\
\hline Neurofibramatosis Type 1 & 1 \\
\hline Congenital Heart Disease & 3 \\
\hline Peripartum Cardiomyopathy & 3 \\
\hline
\end{tabular}

Apart from the above-mentioned findings, we also observed some differences in CMR results. For example, some reports did not include data such as where the noncompaction pattern was most prominent, the ratio of the thickness of the noncompacted and compacted regions which were crucially important for the diagnosis of NCCM. There was also limited information about the trabeculated mass, and LGE.

In our study, an increase in trabeculation was observed mostly in the lateral wall and apex, respectively similar to other studies related to $\operatorname{NCCM}^{(12,13)}$.

CMR imagings of these patients in the study period were evaluated by two radiologists. There are also differences in these reports according to the radiologists even in similar years when evaluations were performed. However, as the years progressed, the reports were observed to be more detailed especially in terms of supporting or excluding the diagnosis. Besides, in the reports that have been evaluated in recent years, it has been observed that consensus among radiologists has become evident.

\section{Discussion}

Although NCCM is classified as primary cardiomyopathy in the 2006 AHA classification, there is still no consensus regarding the etiology, pathophysiology, diagnosis, and treatment of the disease ${ }^{(11)}$.

Echocardiography is the first-line technique in the diagnosis of LVNC and there are different criteria. The most commonly used criterion is Jenni criterion. The measurements were as follows; Demonstration of a twolayer structure on the short axis at the end of systole, the ratio of $\mathrm{NC}$ to $\mathrm{C}$ layer in the section with the most trabeculation is 2.3 and above, showing the reverse and flat blood flow between the trabecular indentations, and the LV cavity ${ }^{(14)}$. The specificity of the existing echocardiography criteria is low ${ }^{(15)}$. In the studies of Rajdev et al. ${ }^{(16)}$ and Caselli et al. ${ }^{(17)}$, it has been shown that the trabeculation network with 3-dimensional (3D) echocardiography has been demonstrated in a better resolution. For this reason, 
it is stated that $3 \mathrm{D}$ echocardiography can be used in differential diagnosis.

CMR is at the forefront in the diagnosis of NCCM because it gives more detailed information about myocardial morphology ${ }^{(18)}$. There are also different criteria regarding CMR imaging. Petersen criterion is the most frequently used criterion, based on the ratio of thicknesses of NC and C layers in the end-diastolic phase to be 2.3 and above $^{(11)}$. The Jacquier criterion that evaluates from another perspective is based on the fact that the trabeculated LV mass is at least $20 \%$ of the global LV mass ${ }^{(12)}$. The new criterion established by Grothoff et al. ${ }^{(19)}$ by modifying the Jacquier criteria provides high sensitivity and specificity in distinguishing NCCM from other diseases with increased trabeculation. In this criterion, trabeculated areas are examined one by one after the removal of blood pool in the noncompacted layers and there are four basic measures: $\mathrm{NC}$ area mass is at least $25 \%$ of the total mass, $\mathrm{NC}$ area mass is above $15 \mathrm{~g} / \mathrm{m}^{2}$, the ratio of $\mathrm{NC}$ and $\mathrm{C}$ area is 2.1, and above in at least one of the $4^{\text {th }}$ to $6^{\text {th }}$ segments and 3.1, and above in at least one of the other segments ${ }^{(20)}$.

There are also strain studies including echocardiography and CMR imaging in recent years ${ }^{(21)}$.

In our study, it was observed that mainly Peterson criterion was used as diagnostic criteria. Jacquier criterion was also used in some CMR reports.

\section{Study Limitations}

The limitations of the study can be listed as the evaluation of the CMR examinations by two different operators, situations encountered due to the examination of reports belonging to a long time and insufficient data concerning diagnostic criteria of NCCM.

\section{Conclusion}

It is still unclear if LVNC would represent a distinct cardiomyopathy or a morphological trait common to different types of cardiomyopathy ${ }^{(4,18,22)}$. Also, other physiological and pathological conditions leading to increase trabeculation should be kept in mind for differential diagnosis ${ }^{(3-5)}$. A clear consensus regarding diagnosis has not been established in this uncertainty. However, as observed in our study, the diagnostic criteria become clearer and standardized over the years.

There is still a need for further investigations about diagnostic imaging modalities and accompanying conditions for the purpose of revealing LVNC clearly.

\section{Ethics}

Ethics Committee Approval: This study approved by local ethical committee at Ege University Faculty of Medicine (date: 8/8/17, decision no: 17-6/12).

Informed Consent: Ethics committee approval was obtained to investigate patients diagnosed with NCCM and data were used from the hospital information system without disclosing patient identities.

Peer-review: Externally peer-reviewed.

Financial Disclosure: The author declared that this study received no financial support.

\section{References}

1. Ritter M, Oechslin E, van der Loo B. Isolated ventricular non-compaction of the myocardium in adults. Heart 2007;93:11-5.

2. Samsa LA, Yang B, Liu J. Embryonic cardiac chamber maturation: trabeculation, conduction and cardiomyocyte proliferation. Am J Med Genet C Semin Med Genet 2013;163C:157-68.

3. Hofer M, Stöllberger C, Finsterer J. Acquired noncompaction associated with myopathy. Int J Cardiol 2007;121:296-7.

4. Arbustini E, Favalli V, Narula N, Serio A, Grasso M. Left Ventricular Noncompaction A Distinct Genetic Cardiomyopathy? State-The-Art-OfReview J Am Coll Cardiol 2016;68:949-66.

5. Oechslin E, Jenni R. Nosology of noncompaction cardiomyopathy: the emperor still wears clothes! Can J Cardiol 2017;33:701-4.

6. Report of the WHO/ISFC task force on the definition and classification of cardiomyopathies. Br Heart J 1980;44:672-3.

7. Vizzardi E, Nodari S, Metra M, Dei Cas L. Non-compaction of the ventricular myocardium Heart Int 2006;2:178.

8. Gati S, Chandra N, Bennett RL, et al. Increased left ventricular trabeculation in highly trained athletes: do we need more stringent criteria for the diagnosis of left ventricular non-compaction in athletes? Heart 2013;99:401-8.

9. D’Ascenzi F, Pelliccia A, Natali BM, Bonifazi M, Mondillo S. Exerciseinduced left-ventricular hypertrabeculation in athlete's heart. Int J Cardiol 2015;181:320-2. 
10. Chebrolu LH, Mehta AM, Nanda NC Noncompaction cardiomyopathy: The role of advanced multimodality imaging techniques in diagnosis and assessment Echocardiography 2017;34:279-89.

11. Maron BJ, Towbin JA, Thiene G, et al. Contemporary definitions and classification of the cardiomyopathies: an American Heart Association Scientific Statement from the Council on Clinical Cardiology, Heart Failure and Transplantation Committee; Quality of Care and Outcomes Research and Functional Genomics and Translational Biology Interdisciplinary Working Groups; and Council on Epidemiology and Prevention. Circulation 2006;113:1807-16

12. Petersen SE, Selvanayagam JB, Wiesmann F, et al. Left Ventricular noncompaction: insights from cardiovascular magnetic resonance imaging. $\mathrm{J}$ Am Coll Cardiol 2015;46:101-5.

13. Jacquier A, Thuny F, Jop B, et al. Measurement of trabeculated left ventricular mass using cardiac magnetic resonance imaging in the diagnosis of left ventricular non-compaction. Eur Heart J 2010;31:1098-104

14. Oechslin E, Jenni R. Left Ventricular Noncompaction From Physiologic Remodeling to Noncompaction Cardiomyopathy J Am Coll Cardiol $2017 ; 12.031$.

15. Niemann M, Störk S, Weidemann F. Left ventricular noncompaction cardiomyopathy: an overdiagnosed disease. Circulation 2012;126:e240-3.

16. Rajdev S, Singh A, Nanda NC, Baysan O, Hsiung MC. Comparison of twoand three-dimensional transthoracic echocardiography in the assessment of trabeculations and trabecular mass in left ventricular noncompaction. Echocardiography 2007;24:760-7.

17. Caselli S, Autore C, Serdoz A, et al. Three-dimensional echocardiographic characterization of patients with left ventricular noncompaction. J Am Soc Echocardiogr 2012;25:203-9.

18. Thuny F, Jacquier A, Jop B, et al. Assessment of left ventricular noncompaction in adults: a side-by-side comparison of cardiac magnetic resonance imaging with echocardiography. Arch Cardiovasc Dis 2010;103:150-9.

19. Grothoff M, Pachowsky M, Hoffmann J, et al. Value of cardiovascular MR in diagnosing left ventricular non-compaction cardiomyopathy and in discriminating between other cardiomyopathies. Eur Radiol 2012;22:2699709.

20. Positano V, Meloni A, Macaione F, et al. Non-compact myocardium assessment by cardiac magnetic resonance: dependence on image analysis method. Int J Cardiovasc Imaging 2018;34:1227-38.

21. Zheng T, Ma X, Li S, et al. Value of Cardiac Magnetic Resonance Fractal Analysis Combined with Myocardial Strain in Discriminating Isolated Left Ventricular Noncompaction and Dilated Cardiomyopathy. Journal of Magnetic Resonance Imaging 2019;50:153-63.

22. Weir-McCall JR, Yeap PM, Papagiorcopulo C, et al. Left ventricular noncompaction: Anatomical Phenotype or Distinct Cardiomyopathy J Am Coll Cardiol 2016;68:2157-65. 